\title{
Effect of 17ß-estradiol or alendronate on the bone densitometry, bone histomorphometry and bone metabolism of ovariectomized rats
}

L.H.B.C. da Paz ${ }^{1}$, V. de Falco ${ }^{1}$, N.C. Teng ${ }^{2}$, L.M. dos Reis ${ }^{2}$, R.M.R. Pereira ${ }^{1}$ and $\mathrm{V}$. Jorgetti2

\author{
Departamentos de ${ }^{1}$ Reumatologia and ${ }^{2} \mathrm{~N}$ efrologia, \\ Faculdade de Medicina, U niversidade de São Paulo, \\ São Paulo, SP, Brasil
}

\section{Correspondence \\ L.H.B.C. da Paz \\ Departamento de Reumatologia \\ Faculdade de Medicina, USP \\ Av. Dr. Arnaldo, 455, 3 andar \\ 01246-930 São Paulo, SP \\ Brasil \\ Fax: + 55-11-3088-7626 \\ E-mail: vandajor@usp.br or \\ Ihemrbcp@usp.br}

Research supported in part by FAPESP (No. 96/07001-5).

Received January 4, 2001 Accepted May 17, 2001

\section{Abstract}

The objective of the present study was to evaluate the effect of 17ßestradiol or alendronate in preventing bone loss in 3-month-old ovariectomized Wistar rats. One group underwent sham ovariectomy (control, $\mathrm{N}=10$ ), and the remaining three underwent double ovariectomy. One ovariectomized group did not receive any treatment $(\mathrm{OVX}, \mathrm{N}=$ 12). A second received subcutaneous $17 \beta$-estradiol at a dose of $30 \mu \mathrm{g} /$ $\mathrm{kg}$ for 6 weeks (OVX-E, $\mathrm{N}=11)$ and a third, subcutaneous alendronate at a dose of $0.1 \mathrm{mg} / \mathrm{kg}$ for 6 weeks (OVX-A, $\mathrm{N}=8)$. Histomorphometry, densitometry, osteocalcin and deoxypyridinoline measurements were applied to all groups. After 6 weeks there was a significant decrease in bone mineral density (BMD) at the trabecular site (distal femur) in OVX rats. Both alendronate and 17ß-estradiol increased the BMD of ovariectomized rats, with the BMD of the OVX-A group being higher than that of the OVX-E group. Histomorphometry of the distal femur showed a decrease in trabecular volume in the untreated group (OVX), and an increase in the two treated groups, principally in the alendronate group. In OVX-A there was a greater increase in trabecular number. An increase in trabecular thickness, however, was seen only in the OVX-E group. There was also a decrease in bone turnover in both OVX-E and OVX-A. The osteocalcin and deoxypyridinoline levels were decreased in both treated groups, mainly in OVX-A. Although both drugs were effective in inhibiting bone loss, alendronate proved to be more effective than estradiol at the doses used in increasing bone mass.

\section{Introduction}

Estrogen deficiency as a consequence of menopause causes osteopenia in about twothirds of women. Thus, estrogen replacement is commonly used as a prophylactic and therapeutic measure. Despite the beneficial effects of estrogen on bone mass, many patients

\author{
Key words \\ - Rat \\ - 17ß-Estradiol \\ - Alendronate \\ - Densitometry \\ - Histomorphometry
}

refuse estrogens for various reasons (1).

Alendronate, like other bisphosphonate compounds, can inhibit bone loss by suppressing osteoclastic bone resorption, and has been studied extensively for the treatment of human osteoporosis (2). There are several hypotheses attempting to explain its action on bone resorption (3-12). Among the 
different animal models (13-16) and methods (17) applied in studies inducing osteoporosis, the model most frequently used is the ovariectomized rat (18-21). This model allows us to study the bone loss and to evaluate the effects of drugs commonly used to treat osteoporosis.

Although there have been many clinical and experimental studies on the effects of estrogen or bisphosphonate on osteoporosis, the present study is one of the first to analyze the effects of both drugs at the same time by means of invasive and noninvasive methods. The aim of the present study was to compare the effects of 17ß-estradiol and the bisphosphonate alendronate in preventing bone loss in ovariectomized rats. To determine the effects of the drug, we measured bone mineral density (BMD), performed histomorphometric analysis of the distal femur and measured biochemical markers of bone turnover.

\section{Material and Methods}

\section{Animals}

Three-month-old female Wistar rats were maintained under constant conditions of temperature $\left(20 \pm 1^{\circ} \mathrm{C}\right)$ and light (12-h light-dark cycle) with ad libitum access to food and water.

\section{Surgical procedures}

The rats were sham operated or underwent bilateral ovariectomy after being anesthetized with ketamine (Ketalar, Parke-Davis, Buenos Aires, Argentina) and Xylazine (Rompum, Bayer, São Paulo, SP, Brazil). In the ovariectomized rats a ventral incision was made to expose the ovaries which were removed after ligation of the uterine horn.

\section{Protocol}

The following groups were formed: shamoperated control rats $(\mathrm{N}=10)$, ovariecto- mized rats receiving saline only $(\mathrm{OVX}, \mathrm{N}=$ 12), ovariectomized rats receiving $17 ß-e s-$ tradiol (Sigma Chemical Co., St. Louis, MO, USA) dissolved in small amounts of ethanol with the volume adjusted with olive oil to give a concentration of $30 \mu \mathrm{g} / \mathrm{kg}$ body weight and administered daily subcutaneously for 6 weeks (OVX-E, N=11), ovariectomized rats receiving alendronate (Merck Sharp and Dohme, Ranway, NJ, USA) dissolved in saline and administered daily subcutaneously for 6 weeks at a dose of $0.1 \mathrm{mg} /$ $\mathrm{kg}$ body weight (OVX-A, $\mathrm{N}=8)$. All rats were sacrificed after 6 weeks. On the 2nd, $3 \mathrm{rd}, 28 \mathrm{th}$, and 29th days prior to sacrifice, they received oxytetracycline (Terramycin, Pfizer, Guarulhos, São Paulo, Brazil) administered intramuscularly at a dose of $20 \mathrm{mg} / \mathrm{kg}$ for bone labeling. Femora were then obtained for mineralized bone histology and histomorphometry.

\section{Bone mineral measurements}

BMD was measured by dual-energy Xray absorptiometry (DXA; Hologic QDR2000, Bedford, MA, USA) adapted to the measurement of BMD in small animals. A distal femur scan was performed.

In vivo reproducibility was evaluated by measuring the coefficient of variation $(\mathrm{CV}=$ $100 \times \mathrm{SD} /$ mean) of five BMD measurements in one rat weighing $220 \mathrm{~g}$, each time repositioning the rat at the two different sites. The variation was $1.4 \%$ in distal femur. All parameters were measured twice, i.e., at baseline and after 6 weeks.

\section{Histomorphometry}

The distal right femur was fixed in $70 \%$ ethanol, dehydrated, embedded in methylmethacrylate, and sectioned longitudinally using a Policut S microtome (Reichert-Jung, Heidelberg, Germany). We obtained 5- and $10-\mu \mathrm{m}$ sections from the center of each specimen. The $5-\mu \mathrm{m}$ section was stained with 
$0.1 \%$ toluidine blue, $\mathrm{pH} 6.4$, and at least two nonconsecutive sections were examined for each sample. Static and structural parameters of bone formation and resorption were measured at a standardized site below the growth plate in the secondary spongiosa using a semi-automatic method (Osteometrics, Inc., Atlanta, GA, USA). Kinetic data were obtained by means of a Zeiss integrating eyepiece II or a calibrated eyepiece. Kinetic bone parameters were obtained from unstained $10-\mu \mathrm{m}$ sections examined by fluorescent light microscopy (Nikon, Tokyo, Japan). All histomorphometric indices were reported according to the standardized nomenclature recommended by the American Society of Bone and Mineral Research (22). All animal data were obtained by blind measurements.

\section{Blood and urine collection and assays}

Urine was collected in metabolic cages. Urinary deoxypyridinoline (Dpyr) was measured by ELISA (Metra Biosystems, Palo Alto, CA, USA) and creatinine with a Covas-Integra Auto Analyzer (Roche, Branchburg, NJ, USA). The rats were then sacrificed by exsanguination while under ether anesthesia. Serum osteocalcin was also measured by ELISA (Biomedical Technologies Inc., Stoughton, MA, USA).

\section{Statistical analysis}

Data are reported as mean \pm standard deviation (SD). The paired Student $t$-test was used to analyze values within the same group at baseline and after 6 weeks. ANOVA followed by the Newman-Keuls post-test was used to compare different groups. Linear regression between histomorphometric variables and noninvasive bone mass measurements was calculated and the Pearson test was applied.

Statistical significance was set at $\mathrm{P}$ values lower than 0.05 .

\section{Results}

\section{Body weight}

All rats in the study gained weight over the 6-week experimental period and there was no significant difference between the four groups at baseline or after 6 weeks, as shown by the following weight values: control: $221.1 \pm 10.1$ and $265.0 \pm 24.2 \mathrm{~g}$, OVX: $222.0 \pm 13.6$ and $271.8 \pm 24.5$ g, OVX-E: $228.7 \pm 8.6$ and $255.6 \pm 13.7 \mathrm{~g}$, and OVX-A: $222.5 \pm 10.9$ and $254.8 \pm 24.3 \mathrm{~g}$.

\section{Bone mineral density}

No significant differences in baseline BMD were observed between groups. After 6 weeks, no significant difference was observed in the control group compared with baseline; however, a remarkable BMD decrease was observed in the distal femur of the OVX group. A significant increase in BMD was observed in the OVX-A group and a nonsignificant increase was observed in the OVX-E group. At the end of the experimental period, BMD was significantly lower in OVX and higher in OVX-A than in the other groups (Figure 1).

\section{Histomorphometry}

The static histomorphometric parameters of the distal femur showed lower trabecular bone volume (BV/TV) and trabecular number

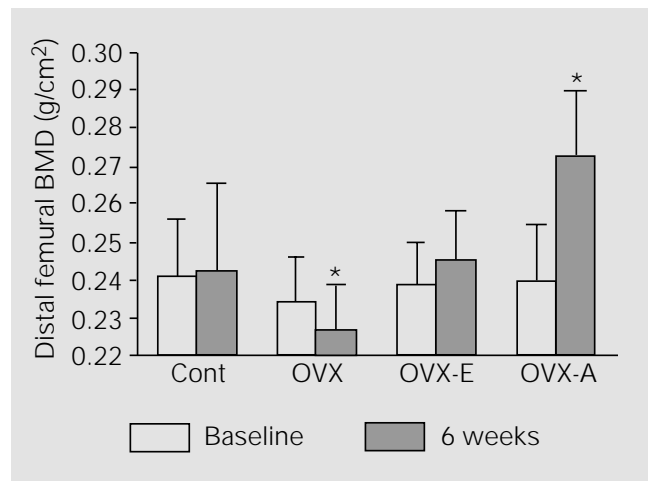

Figure 1. Bone mineral density (BMD) measured by dual-energy X-ray absorptiometry of the distal femur in the four groups: control (Cont), ovariectomized (OVX), ovariectomized treated with $17 ß$ estradiol for 6 weeks (OVX-E), and ovariectomized treated with alendronate for 6 weeks (OVXA). Data are reported as the mean \pm SD. Difference between 6 weeks and baseline. ${ }^{*} \mathrm{P}<0.05$ compared to respective baseline (Student t-test). 
in OVX compared with control. This reduction was significantly inhibited by 173 -estradiol and alendronate treatment. The BV/TV ratio and trabecular number measured in OVXA were significantly higher than in OVX-E (Figure 2). In contrast, trabecular thickness was higher in OVX-E than in OVX-A.

Figure 2. Bone volume and trabecular number in the distal femur in the four groups: sham control (Cont), ovariectomized (OVX), ovariectomized treated with 17ß-estradiol for 6 weeks (OVX-E), and ovariectomized treated with alendronate for 6 weeks (OVX-A). Data are reported as means $\pm \mathrm{SD}$. $\mathrm{P}<0.05$ : *vs OVX; " VS OVX-A; +vs control (ANOVA).

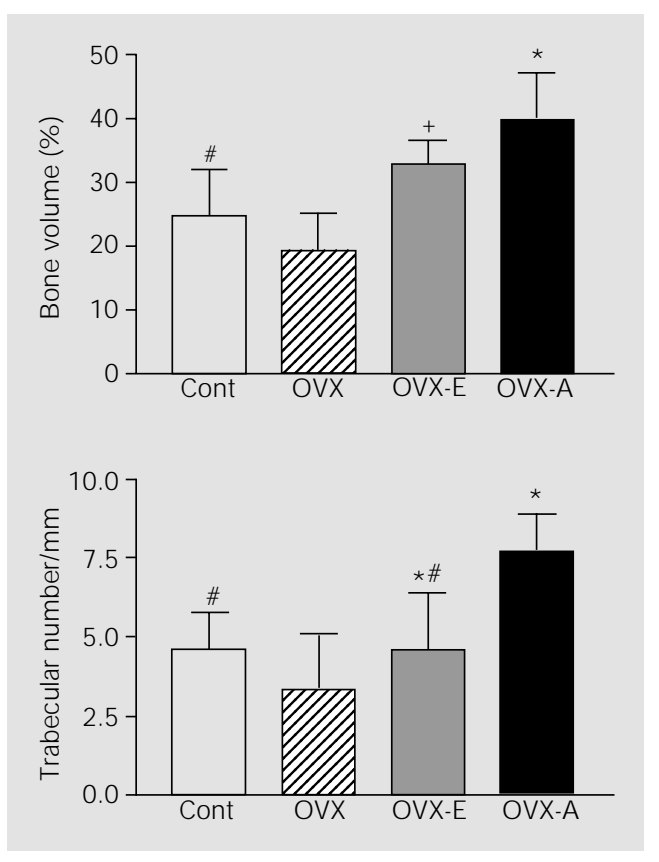

The OVX group presented signs of high bone turnover indicated by elevated osteoid volume and surface, increased erosion, and increased osteoblast and osteoclast surface without an increase in mineral apposition rate.

Treatment with $17 ß$-estradiol and alendronate produced a reduction in bone turnover mainly by changes in bone formation parameters. This effect was particularly remarkable in the animals receiving alendronate. No mineralization impairment was noted in the alendronate group (Table $1)$.

\section{Biochemistry}

Bone resorption and formation markers measured by determination of Dpyr crosslinks and osteocalcin, respectively, were increased in the OVX group compared to control, but the difference was significant only in the osteocalcin assay. The 17ß-estradiol and alendronate treatments were associated with reduced Dpyr cross-link excretion and serum osteocalcin when compared to ovariectomized untreated rats (Figure 3).

Table 1. Histomorphometric variables of trabecular bone in the distal femur.

\begin{tabular}{lccccr}
\hline & Cont $(\mathrm{N}=10)$ & OVX $(\mathrm{N}=12)$ & OVX-E $(\mathrm{N}=11)$ & OVX-A $(\mathrm{N}=8)$ & P value \\
\hline BV/TV $(\%)$ & $24.8 \pm 7.2^{* \#}$ & $19.4 \pm 5.8$ & $32.8 \pm 3.9^{* \#+}$ & $40.0 \pm 7.3^{*}$ & $<0.0001$ \\
Tb.Th $(\mu \mathrm{m})$ & $53.7 \pm 8.9$ & $58.1 \pm 6.3$ & $63.3 \pm 4.8$ & $52.1 \pm 8.8$ & 0.0137 \\
Tb.Sp $(\mu \mathrm{m})$ & $181.1 \pm 79.3^{*} \#$ & $262.8 \pm 87.1$ & $131.0 \pm 18.0^{* \#}$ & $80.5 \pm 21.1^{*}$ & $<0.0001$ \\
Tb.N/mm & $4.6 \pm 1.2^{* \#}$ & $3.3 \pm 0.8$ & $4.6 \pm 1.8^{*}$ & $7.7 \pm 1.2^{*}$ & $<0.0001$ \\
Es/Bs $(\%)$ & $6.6 \pm 4.2$ & $10.1 \pm 7.3$ & $8.3 \pm 2.8$ & $8.0 \pm 3.6$ & 0.5180 \\
Oc.s/Bs $(\%)$ & $2.2 \pm 2.0$ & $2.4 \pm 2.4$ & $1.6 \pm 0.8$ & $1.8 \pm 0.8$ & 0.7470 \\
OV/BV $(\%)$ & $0.2 \pm 0.2^{*}$ & $0.6 \pm 0.4$ & $0.2 \pm 0.1^{*}$ & $0.03 \pm 0.03^{*}$ & $<0.0001$ \\
O.Th $(\mathrm{mm})$ & $2.6 \pm 0.7$ & $3.0 \pm 1.1$ & $2.8 \pm 0.7$ & $1.6 \pm 1.3$ & 0.3147 \\
Os/Bs $(\%)$ & $2.3 \pm 1.2^{*}$ & $6.1 \pm 4.4$ & $2.1 \pm 1.4^{*}$ & $0.4 \pm 0.4^{*}$ & 0.0002 \\
Ob.s/Bs $(\%)$ & $1.2 \pm 0.8^{*}$ & $3.6 \pm 2.8$ & $1.6 \pm 1.3^{*}$ & $0.3 \pm 0.3^{*}$ & 0.0014 \\
MAR $(\mu \mathrm{m} /$ day) & $0.3 \pm 0.1^{*}$ & $0.2 \pm 0.1$ & $0.1 \pm 0.0^{*}$ & $0.2 \pm 0.0^{*}$ & 0.0004 \\
& & & & &
\end{tabular}

Cont, sham-operated control rats; OVX, untreated ovariectomized rats; OVX-E, ovariectomized rats + 17ßestradiol; OVX-A, ovariectomized rats + alendronate. Bone histomorphometric variables for the distal femur are abbreviated as follows: BV/TV = trabecular bone volume; $\mathrm{Tb} . \mathrm{Th}=$ trabecular thickness; Tb.Sp = trabecular separation; Tb.N = trabecular number; Es/Bs = eroded surface; Oc.s/Bs = osteoclastic surface; OV/BV = osteoid volume; O.Th = osteoid thickness; Os/Bs = osteoid surface; Ob.s/Bs = osteoblastic surface; MAR = mineral apposition rate. *vs OVX; "*vs OVX-A; +vs Cont. 


\section{Correlation between BMD and histomorphometric parameters}

BMD measurements at the distal femur were positively correlated with BV/TV and trabecular number. In contrast, BMD was negatively correlated with trabecular separation (Figure 4).

\section{Discussion}

Bone loss induced by ovariectomy in rats has been widely used as a model of postmenopausal osteoporosis and has been validated as a clinically relevant model of this condition in humans. In our study, bone loss induced by ovariectomy was observed in untreated rats 6 weeks after extraction. The site imaged was the distal femur metaphysis, an area that represents cancellous bone and loses bone rapidly after ovariectomy. Several studies have described similar findings
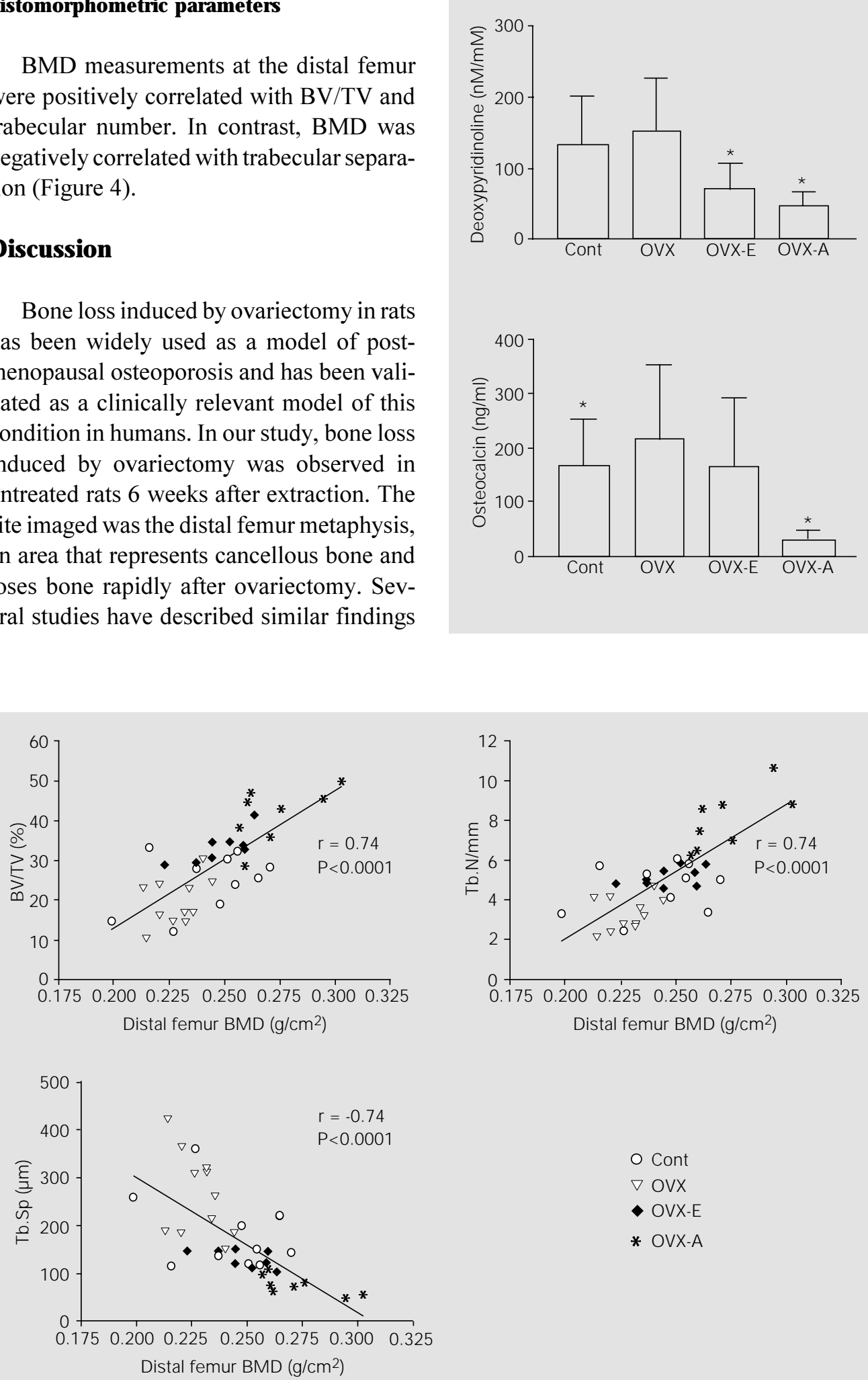

Figure 3. Deoxypyridinoline and osteocalcin measured by ELISA during the 6th week: control (Cont), ovariectomized (OVX), ovariectomized treated with $17 ß$-estradiol for 6 weeks (OVX$\mathrm{E})$, and ovariectomized treated with alendronate for 6 weeks (OVX-A). Bars represent the mean $\pm S D$. $* P<0.05$ compared to OVX (ANOVA).
Figure 4. Correlation between distal femur bone mineral density (BMD) determined by dualenergy X-ray absorptiometry and trabecular volume (BV/TV), trabecular number (Tb.N) and trabecular separation (Tb.Sp) in the distal femur. Cont, sham-operated control rats; OVX, untreated ovariectomized rats; OVX-E, ovariectomized rats +17 ß-estradiol; OVX-A, ovariectomized rats + alendronate. 
related to estrogen deficiency lasting a few days to several weeks $(23,24)$.

We chose invasive and noninvasive techniques to analyze changes caused by ovariectomy and the effects of $17 ß$-estradiol and alendronate. There has been little documentation analyzing correlations between bone parameters using these techniques in rats $(25,26)$. In the present study, distal femur BMD was correlated with histomorphometric parameters. Although histomorphometry has higher resolution and permits the quantification of both dynamic and static bone parameters, DXA demonstrated practical advantages by permitting longitudinal skeletal quantification in vivo (27-31). Our analysis confirms that both methods are indeed useful for the measurement of bone.

Histomorphometric analysis confirmed that alendronate increased bone mass more effectively than $17 ß$-estradiol. An interesting finding was that both groups showed higher bone mass restoration than the nonovariectomized group. Similar findings were observed by other authors. Seedor et al. (32) administered alendronate and observed a bone mass increase. The same findings were obtained by Belena et al. (33) in nonhuman primates. Similarly, Kalu et al. (34) administered 17ß-estradiol to ovariectomized animals and found an increase in BV/TV compared to the non-ovariectomized group. Tobias and Compston (35) suggested that estrogen can stimulate osteoblast function and perhaps perform an anabolic action. They suggested that antireabsorptive drugs such as estrogen would be interesting to use in high doses in certain relatively short-term situations, such as the development of osteoporosis in the early postmenopausal period.

Other studies have shown that these drugs were effective in increasing BV/TV; however, comparisons cannot be made with our protocol due to the great variation in doses and average treatment time used in these trials. In our study, we have used minimum doses with maximum effects $(32,36,37)$. There is no correlation between doses in the present study and in humans.

We believe that the bone loss observed with acute estrogen deficiency represents the increase in remodeling space that occurs when the bone turnover rate accelerates. In the treatment with estrogen or bisphosphonates there is generally a reduction of bone turnover to premenopausal levels or below, when assessed by histomorphometric measurements. This decrease is thought to be associated with the maintenance of bone mass in postmenopausal women and in animal studies. Our findings agree with those reported by others $(16,20,23,26,36)$.

Alendronate had no significant effect on histomorphometric bone resorption parameters, including eroded surface and osteoclast surface. In contrast, alendronate significantly inhibited urinary excretion of Dpyr. The absence of a decrease in osteoclastic surface suggests reduction of bone resorption through a slowing-down function $(4,38)$, contrary to other studies which have suggested a decrease in osteoclastogenesis or an increase in osteoclast apoptosis $(7,19)$.

Mineralization defects have been reported in etidronate, the first bisphosphonate used for osteoporosis treatment (36). High doses of alendronate, however, did not produce increased surface, volume or osteoid thickness, nor did they significantly change the mineral apposition rate.

Furthermore, ovariectomy was also associated with an increase in serum osteocalcin and urinary Dpyr cross-link excretion. Both $17 ß$-estradiol and alendronate treatments were associated with suppression of these parameters to values below that observed in the controls, reflecting an important reduction of bone remodeling primarily in rats treated with alendronate. This drastic decrease in bone turnover could also explain the important increase in bone mass. These findings are consistent with those of Delmas et al. (39) who reported a positive correla- 
tion between the biochemical parameters (e.g., pyridinoline) and histomorphometric parameters measured on the iliac crest in patients with osteoporosis.

Because of the little documentation available comparing 17ß-estradiol and alendronate in preventing bone loss in the same experiment, our study has proven to be an interesting investigative experiment. BMD analysis demonstrated that treatment with high doses of 17ß-estradiol and alendronate prevents ovariectomy-induced bone loss in female rats. We encountered only one study that compared the effects of both drugs in the same experiment. Lumbar and proximal tibiae were examined by computed tomography and by histomorphometry. The authors observed that $17 \AA$-estradiol was more effective than alendronate at the doses of $0.03 \mathrm{mg} / \mathrm{kg}$, subcutaneously, twice weekly and $100 \mu \mathrm{g} /$ $\mathrm{kg}$, orally, respectively (26).

We concluded that at the doses used in the present study, both drugs are effective in preventing bone loss after ovariectomy with alendronate producing a better outcome when compared with $17 ß$-estradiol.

\section{Acknowledgments}

The authors are grateful to Mr. Wagner Vasques Dominguez for statistical assistance.

\section{References}

1. Lindsay R (1995). Estrogen deficiency. In: Riggs BL \& Melton III LJ (Editors), Osteoporosis: Etiology, Diagnosis and Management. Raven Press, New York.

2. Kanis J A, Gertz BJ , Singer F \& Ortolani S (1995). Rationale for the use of alendronate in osteoporosis. Osteoporosis International, 5: 1-13.

3. BeekEV, Lowik C, Pluijm GDP \& Papoulos $S$ (1999). The role of geranylgeranylation in bone resorption and its suppression by bisphosphonates in fetal bone. Explants in vitro: a clue to the mechanism of action of nitrogen-containing bisphosphonates. J ournal of Bone and Mineral Research, 14: 722-729.

4. Colucci S, Minielli V, Zambonin G, Cirulli N, Mori G, Serra M, Patella V, Zallone AZ $\&$ Grano M (1998). Alendronate reduces adhesion of human osteoclast-like cells to bone and bone protein-coated surfaces. Calcified Tissue International, 63: 230235.

5. Giuliani N, Pedrazzoni M, Passeri G \& Girasole $G$ (1998). Bisphosphonates inhibit IL-6 production by human osteoblast-like cells. Scandinavian J ournal of Rheumatology, 27: 38-41.

6. Ito M, Amizuka N, Nakajima T \& Ozawa H (1999). Ultrastructural and cytochemical studies on cell death of osteoclasts induced by bisphosphonate treatment. Bone, 25: 447-452.

7. Nishikawa M, Akatsu T, Katayama $Y$, Yasutomo Y, Kado S, Kugai N, Yamamoto
M \& Nagata N (1996). Bisphosphonates act on osteoblastic cells and inhibit osteoclast formation in mouse marrow cultures. Bone, 18: 9-14.

8. Plotkin $\mathrm{LI}$, Weinstein RS, Parfitt AM, Roberson PK, Manologas SC \& Bellido T (1995). Prevention of osteocyte and osteoblast apoptosis by bisphosphonates and calcitonin. J ournal of Clinical Investigation, 104: 1363-1374.

9. Rodan GA \& Fleisch HÁ (1996). Bisphosphonates: Mechanisms of action. J ournal of Clinical Investigation, 97: 2692-2696.

10. Sahni M, Guenther HL, Fleisch $H$, Collin $P$ $\&$ Martin J (1993). Bisphosphonates act on rat bone resorption through the mediation of osteoblasts. J ournal of Clinical Investigation, 91: 2004-2011.

11. Sato M, Grasser W, Endo N, Akins R, Simmons H, Thompson DD, Golub E \& Rodan GA (1991). Alendronate localization in rat bone and effects on osteoclast ultrastructure. J ournal of Clinical Investigation, 88: 2095-2105.

12. Vitté C, Fleisch H \& Guenther HL (1996). Bisphosphonates induce osteoblasts to secrete an inhibitor of osteoclast-mediated resorption. Endocrinology, 137: 2324-2333

13. Miller SC \& Bowman Jee WSS (1995). Available animal models of osteopenia small and large. Bone, 17 (Suppl 4): 117123.

14. Mosekilde LI (1995). Assessing bone quality animal models in preclinical osteoporo- sis research. Bone, 17 (Suppl 4): 343-352.

15. Rodgers BJ , Faugere MCM \& Malluche $\mathrm{H}$ (1993). Animal models for the study of bone loss after cessation of ovarian function. Bone, 14: 369-377.

16. Thompson DD, Simmons HA, Pirie CM \& $\mathrm{Ke} \mathrm{HZ} \mathrm{(1995).} \mathrm{FDA} \mathrm{guidelines} \mathrm{and} \mathrm{animal}$ models for osteoporosis. Bone, 17 (Suppl 4): 125-133.

17. Mohamed S, Shapira D, Leichter I, Reznick A \& Silbermann M (1998). Ability of different techniques of measuring bone loss in aging female rats. Calcified Tissue International, 42: 375-382.

18. Frost HM \& J ee WSS (1991). On the rat model of human osteopenias and osteoporosis. Bone and Mineral, 18: 227 236.

19. Kalu DN (1991). The ovariectomized rat model of postmenopausal bone loss. Bone and Mineral, 15: 175-192.

20. Wronski TJ, Lowry PL, Walsh CC \& Ignaszewski A (1985). Skeletal alterations in ovariectomized rats. Calcified Tissue International, 37: 324-328.

21. Yamazaki I \& Yamaguchi H (1989). Characteristics of an ovariectomized osteopenic rat model. J ournal of Bone and M ineral Research, 4: 13-22.

22. Parfitt $A M$, Drezner $M K$, Glorieux $F H$, Kanis J A, Malluche $\mathrm{H}$, Meunier PJ $\mathrm{H}$, Ott SM \& Recker RR (1987). Bone histomorphometry: Standardization of nomenclature, symbols, and units. J ournal of Bone and Mineral Research, 2: 595-610. 
23. Lane NE, Haupt D, Kimmel DB, Modin G \& Kinney JH (1999). Early estrogen replacement therapy reverses the rapid loss of trabecular bone volume and prevents further deterioration of connectivity in the rat. J ournal of Bone and Mineral Research, 14: 206-214.

24. Pastoriau P, Chomel A \& BonnetJ (1995). Specific evaluation of localized bone mass and bone loss in the rat using dual-energy absorptiometry subregional analysis. Osteoporosis International, 5: 143-149.

25. Cosman F, Schnitzer MB, McCann PD, Parisien MV, Dempster DW \& Lindsay R (1992). Relationships between quantitative histological measurements and noninvasive assessments of bone mass. Bone, 13: 237-242.

26. Sato $M, B$ Byant $H U$, Iversen $P$, Helterbrand $\mathrm{J}$, Smietana F, Bemis K, Higgs R, Turner CH, Owan I, Takano Y \& Burr DB (1996). Advantages of raloxifene over alendronate or estrogen on nonreproductive and reproductive tissues in the long-term dosing of ovariectomized rats. J ournal of Pharmacology and Experimental Therapeutics, 279: 298-305.

27. Ammann $P$, Rizzolo $R$, Slosman $D \&$ Bonjour J P (1992). Sequencial and precise in vivo measurement of bone mineral density in rats using dual energy X-ray absorptiometry. J ournal of Bone and M ineral Research, 7: 311-316.

28. Mitlak BH \& Sato M (1997). Bone mineral measurements by DXA in animals. In: Arnett TR \& Henderson B (Editors), Meth- ods in Bone Biology. Chapman \& Hall, London.

29. Griffin MG, Kimble R, Hopper W \& Pacifici R (1993). Dual-energy X-ray absorptiometry of the rat: Accuracy, precision and measurement of bone loss. Journal of Bone and Mineral Research, 8: 795-800.

30. Rozemberg S, Vandromme J, Neve J, Aguilera A, Muregancuro A, Peretz A, KinthaertJ \& Ham H (1995). Precision and accuracy of in vivo bone mineral measurement in rats using dual-energy X-ray absorptiometry. Osteoporosis International, 5: 47-53.

31. Yamaguchi $H$, Kushida $K$, Yamazaki $K$ \& Imove T (1995). Assessment of spine bone mineral density in ovariectomized rats using DXA. J ournal of Bone and Mineral Research, 10: 1033-1039.

32. Seedor J G, Quarruccio HÁ \& Thompson DD (1991). The bisphosphonate alendronate (MK-217) inhibits bone loss due to ovariectomy in rats. J ournal of Bone and Mineral Research, 6: 339-346.

33. Belena R, Toolan BC, Shea M, Markatos A, Meyers ER, Lee SC, Opas EE, Seedor J G, Klein H, Frankenfield D, Quartuccio H, Floravanti C, Clair J, Brown E, Hayes WCM \& Rodan GA (1993). The effects of 2-year treatment with the aminobisphosphonate alendronate on bone metabolism, bone histomorphometry, and bone strength in ovariectomized nonhuman primates. J ournal of Clinical Investigation, 92: 2577-2586.

34. Kalu DN, Liu CC, Salermo E, Hollis B,
Echon R \& Ray M (1991). Skeletal response of ovariectomized rats to low and high doses of 17 ß-estradiol. Bone and Mineral, 14: 175-187.

35. Tobias J H \& Compston J E (1999). Does estrogen stimulate osteoblast function in postmenopausal women? Bone, 24: 121124

36. Wronski TJ, Yen F \& Scott KS (1991). Estrogen and diphosphonate treatment provides long-term protection against osteopenia in ovariectomized rats. J ournal of Bone and Mineral Research, 6: 387394.

37. Wronski TJ, Cintrón M, Doherty AL \& Dann LM (1988). Estrogen treatment prevents osteopenia and depresses bone turnover in ovariectomized rats. Endocrinology, 123: 681-686.

38. Chavassieux PM, Arlot ME, Roux JP, Portero N, Daifotis A, Yates AJ, Hamdy NAT, Malice MP, Freedholm D \& Meunier PJ (2000). Effects of alendronate on bone quality and remodeling in glucocorticoidinduced osteoporosis: A histomorphometric analysis of transiliac biopsies. J ournal of Bone and Mineral Research, 15: 754-762.

39. Delmas PD, Schlemmer A, Gineyts E, Riis B \& Christiansen C (1991). Urinary excretion of pyridinoline crosslinks correlates with bone turnover measured on iliac crest biopsy in patients with vertebral osteoporosis. J ournal of Bone and Mineral Research, 6: 639-646. 